\title{
Molecular mechanisms of the effect of TGF- $\beta 1$ on U87 human glioblastoma cells
}

\author{
IGOR BRYUKHOVETSKIY $^{1}$ and VALERIY SHEVCHENKO ${ }^{1,2}$ \\ ${ }^{1}$ Laboratory of Molecular and Cellular Neurobiology, School of Biomedicine, \\ Far Eastern Federal University, Vladivostok 690091; ${ }^{2}$ Laboratory of Onco Proteomics, \\ NN Blokhin Russian Cancer Research Center of The Ministry of Health of The \\ Russian Federation, Moscow 115478, Russian Federation
}

Received November 25, 2015; Accepted May 26, 2016

DOI: $10.3892 / 01.2016 .4756$

\begin{abstract}
Glioblastoma multiforme (GBM) is the most widespread and aggressive type of primary brain tumor. The prognosis following diagnosis with GBM is poor, with a median survival time of 14 months. Tumor cell invasion, metastasis and proliferation are the major causes of mortality in patients with GBM. In order to develop effective GBM treatment methods it is necessary to identify novel targets involved in these processes. Recently, there has been increasing interest in investigating the signaling pathways involved in GBM development, and the transforming growth factor- $\beta$ (TGF- $\beta$ ) signaling pathway is understood to be significant for regulating the behavior of GBM, as well as stimulating its invasion and metastatic development. Particular interest has been given to investigating the modulation of TGF- $\beta$-induced epithelial-to-mesenchymal transition (EMT); during this process, epithelial cells transdifferentiate into mobile cells with a mesenchymal phenotype. The induction of EMT increases the invasiveness of various types of carcinoma; however, the role of TGF- $\beta$ in this process remains to be elucidated, particularly in the case of GBM. The current study presents a comparative proteome mapping of the U87 human glioblastoma cell line, with and without TGF- $\beta 1$ treatment. Proteome analysis identified numerous proteins involved in the molecular mechanisms of GBM oncogenesis and TGF- $\beta 1$ signaling in glioblastoma. The results of the present study facilitated the identification of novel potential markers of metastasis and candidates for targeted glioblastoma therapy, which may potentially be validated and used in clinical medicine to develop improved approaches for GBM diagnosis and treatment.
\end{abstract}

Correspondence to: Dr Igor Bryukhovetskiy, Laboratory of Molecular and Cellular Neurobiology, School of Biomedicine, Far Eastern Federal University, 8 Sukhanova Street, Vladivostok 690091, Russian Federation

E-mail: igbryukhovetskiy@gmail.com

Key words: glioblastoma multiforme, stem cells, cancer stem cells, transforming growth factor $1 \beta$, proteomic mapping, targeted therapy

\section{Introduction}

Glioblastoma multiforme (GBM) is the most widespread and aggressive type of primary brain tumor (1). Standard treatment strategies are commonly ineffective (2). Even following complex modern treatment regimens, the prognosis for patients with GBM is poor, with a median survival time of 14 months (2). The unsuccessful treatment of this aggressive disease is commonly due to late diagnosis, a lack of specific diagnostic markers, resistance to traditional treatment, and the high proliferation and metastatic potential of tumor cells (3).

The invasion and infiltration of tumor cells, as well as an extremely high proliferation rate, are significant contributors to mortality in patients with GBM (4). In order to develop effective treatment methods, it is necessary to identify novel targets involved in GBM tumorigenesis (4). Thus, there has recently been an increase in interest towards investigating the signaling pathways associated with glioma (5). Ultimately, improving the understanding of the underlying molecular mechanisms of GBM oncogenesis will aid the development of novel and more effective methods of treating the disease.

As one of the various signaling pathways associated with glioma, transforming growth factor- $\beta$ (TGF- $\beta$ ) signaling is important for regulating the behavior of these tumors (5). It has been previously reported that TGF- $\beta$ levels are high in the blood serum and tumor tissue of patients with malignant glioma, and this level was correlated with the type of malignancy, the tumor developmental stage and the patient prognosis (6). Furthermore, it was also hypothesized that the TGF- $\beta$ signaling pathway is directly involved in the molecular mechanisms of glioma malignancy (6). Certain studies have reported that TGF- $\beta$ is able to induce metastatic processes and tumor progression via autocrine mechanisms $(7,8)$.

TGF- $\beta$ is a member of a large cytokine family involved in regulating various biological processes, including fetal development and tissue homeostasis (9). At the molecular level, TGF- $\beta$ affects numerous cellular activities, including proliferation, survival, differentiation, migration and immune activation, depending on the cell type and cellular context (9). TGF- $\beta$ performs its multiple functions via a complex network of various ligands and receptors, which transmit corresponding signals $(9,10)$. Regarding cancer, the TGF- $\beta$ signaling pathway 
exhibits tumor suppressive and oncogenic functions (10). TGF- $\beta$ is considered to be a tumor suppressor as it strongly inhibits the proliferation of epithelial cells, astrocytes and immunocytes (6). Certain tumors avoid the cytostatic response to TGF- $\beta$ via the mutation of particular genes involved in the TGF- $\beta$ signaling pathway (7). Several malignant tumors, including gliomas, selectively block the ability of TGF- $\beta$ to inhibit proliferation, while preserving its other functions (9). In such tumors, TGF- $\beta$ may induce proliferation, angiogenesis, invasion, metastasis and immune suppression. Thus, TGF- $\beta$ is able to exert a dual effect on carcinogenesis depending on the stage and type of tumor; it may act as a tumor suppressor or an oncogene $(11,12)$. This conversion from tumor suppression to oncogenic activity is known as the 'TGF- $\beta$ paradox' $(13,14)$. Whilst significant progress has been made in the understanding of the TGF- $\beta$ signaling pathway, its importance in glioma oncogenesis remains to be elucidated.

Particular interest has been given to investigating the modulation of TGF- $\beta$-induced epithelial-to-mesenchymal transition (EMT). During this process, epithelial cells lose their intercellular connections and apical-basal polarity, reorganize their cytoskeleton, release ECM proteins, and transdifferentiate into mobile mesenchymal cells (15). EMT induction leads to the metastatic invasion of a number of carcinomas; however, the full extent of its influence remains to be elucidated, particularly with regard to glioblastomas (16). Previous studies have attributed EMT to the generation of tumor stem cells $(16,17)$. The underlying molecular mechanisms of TGF- $\beta$-induced EMT require further investigation. However, existing data demonstrates that the TGF- $\beta$ signaling pathway is involved in the late stages of glioma oncogenesis and metastasis development, suggesting it may be an important potential candidate for targeted therapy $(4,17)$.

The present study performed comparative proteome mapping of the U87 human glioblastoma cell line, with and without TGF- $\beta 1$ treatment, using the nano-liquid chromatography-tandem mass spectrometry (LC-MS/MS) method. Proteome analysis identified various proteins associated with TGF- $\beta 1$ regulation of GBM oncogenesis, which may be novel potential markers of metastasis or targets for glioblastoma therapy. The target candidates may potentially be validated and used in the clinic to develop novel approaches for the diagnosis and treatment of GBM, one of the most serious forms of cancer.

\section{Materials and methods}

Preparation of U87 human glioblastoma cells. U87 human glioblastoma cells (American Type Culture Collection, Manassas, VA, USA) were seeded onto a 6-well plate (Corning Incorporated, Corning, NY, USA) at a density of 50,000 cells/well, and cultured in Dulbecco's modified Eagle's medium (DMEM)/F12 (Invitrogen; Thermo Fisher Scientific, Inc., Waltham, MA, USA) supplemented with $10 \%$ fetal calf serum (Invitrogen; Thermo Fisher Scientific, Inc.), 1\% 200 mM L-glutamine (Invitrogen; Thermo Fisher Scientific, Inc.) and $20 \mathrm{mM}$ HEPES (Thermo Fisher Scientific, Inc.), at $37^{\circ} \mathrm{C}$ in a $5 \% \mathrm{CO}_{2}$ humidified atmosphere until $30 \%$ confluence was reached. Subsequently, the cells were washed with Hank's balanced salt solution (Invitrogen; Thermo Fisher Scientific,
Inc.) and serum-free DMEM/F12 with TGF- $\beta 1$ (5 ng/ml) was applied for $72 \mathrm{~h}$.

Monolayer cell cultures were extracted from the 6-well plate, centrifuged at $209 \times \mathrm{g}$ for $10 \mathrm{~min}$ at room temperature, and the supernatant was removed. The cells were resuspended in $3 \mathrm{ml}$ phosphate-buffered saline (PBS; $\mathrm{pH}=7.4$ ) and washed twice more with PBS.

Tumor cell lysates were extracted and low molecular weight compounds were removed using a previously described method (18).

Preparation of samples for mass spectrometry. Following enzymatic cleavage (trypsinolysis) of dried cell lysate samples (18), $4 \mu$ l peptide solution was analyzed via the nano-LC-MS/MS method to confirm successful trypsinolysis. Ammonium bicarbonate was used as LC-MS eluent additive (buffer). Trypsin (Thermo Fisher Scientific) was used for trypsinization, as described previously (18). Following completion of this reaction, the samples were concentrated at $30^{\circ} \mathrm{C}$ in a Labconco CentriVap centrifugal concentrator to remove all ammonium bicarbonate.

Tryptic peptides were diluted during the mobile phase (30\% acetonitrile, $70 \%$ water and $0.1 \%$ formic acid; $\mathrm{pH} 2.7$ ) and divided into 24 fractions using a Dionex UltiMate 3000 chromatograph (Dionex, Sunnyvale, CA, USA) equipped with a fraction collector and MIC-10-CP cation-exchange column (Poros 10S material, $1 \mathrm{~mm}$ x $10 \mathrm{~cm}$; Thermo Fisher Scientific, Inc.) (18). The resulting fractions were concentrated at $30^{\circ} \mathrm{C}$ in the centrifugal concentrator and re-diluted with $100 \mu 10.1 \%$ formic acid.

Mass spectrometry interpretation. The analysis of tryptic peptides was performed using a nano-HPLC Dionex Ultimate 3000 and LTQ Orbitrap XL XM $^{\text {TM }}$ mass spectrometer (Thermo Fisher Scientific, Inc.) with a NanoSpray Ionization source (18). MaxQuant software (version 1.5.2.8; Computational Systems Biochemistry, Max Planck Institute of Biochemistry, Martinsried, Germany) was used for processing mass spectrometry data (19). The table of identified protein families was processed with Perseus software (version 1.5.1.6; Computational Systems Biochemistry, Max Planck Institute of Biochemistry) for annotation, and elimination of contaminating proteins and false positive identifications.

Statistical analysis. Perseus software (version 1.5.1.6) was used to determine statistical differences in the protein levels obtained by the label-free method. Paired samples were compared using Student's t-test. $\mathrm{P}<0.05$ was considered to indicate a statistically significant difference.

\section{Results}

Descriptive analysis. The analysis of nano-LC-MS/MS data with MaxQuant software identified 2,555 proteins in all samples with a high level of accuracy. Additional processing of the data with Perseus software identified the following proteins: Sample01 (U87), 2,336 proteins based on 12,546 peptides; and Sample02 (U87 + TGF- $\beta 1$ ), 2,469 proteins based on 12,707 peptides. Of the total proteins, $71 \%$ were identified based on two or more peptides, and $\sim 29 \%$ based on one 
peptide. The range of the molecular weights of the proteins identified varied between 5.03 and $3,816.00 \mathrm{kDa}$, with 659 proteins weighing $<30 \mathrm{kDa}(1,403$ proteins, $30-100 \mathrm{kDa}$; 446 proteins, $100-300 \mathrm{kDa}$; 35 proteins, 300-500 kDa; and 12 proteins, $>500 \mathrm{kDa}$ ). The coverage percentage of the identified proteins varied from $0.2-95.6 \%$ (1,762 proteins, $20 \%$ coverage; 518 proteins, $20-40 \%$ coverage; 212 proteins, $40-60 \%$ coverage and; 63 proteins, $60-95.6 \%$ coverage). In total, 2,197 proteins ( $~ 86 \%$ of 2,555 proteins) were detected in all cell lysates, 40 were detected only in non-treated U87 cells (58 unique peptides) and 172 were detected in TGF- $\beta 1$-treated U87 cells (219 unique peptides).

The detection sensitivity range, also termed dynamic range, of the mass-spectrometer for the identified proteins was 6-fold (between $4.1 \times 10^{8}$ and 363 ), which allowed for the detection of proteins with low-copy number, including cofilin 2 and tumor necrosis factor (ligand) superfamily, member 13. Additionally, specific markers of mesenchymal (cluster of differentiation (CD)44, integrin $\alpha-5$ and $\beta-1$ ) and progenitor stem cells of glioblastoma (aldehyde dehydrogenase 1 family, member A3), and proneural differentiation (tubulin, $\beta 3$ and nestin) of neural progenitor (nestin) stem cells, were identified.

Comparative bioinformatic analysis was performed on the data obtained from the proteome mapping. All proteins with correlation between normal signal intensities (TGF- $\beta 1$ vs. control) varying between 0.5 and 2 (cutoff value, $<2$-fold change) were not considered during the analysis. Therefore, following TGF- $\beta 1$ stimulation of U87 human glioblastoma cells, in total 635 differentially expressed proteins (DEPs) demonstrated a 2 -fold change of expression $(\mathrm{P}<0.05)$; 512 proteins exhibited increased expression and 123 exhibited decreased expression levels. Of the total 635 identified proteins, 15 exhibited expression level changes $>10$-fold, with 8 significantly increased (immediate early response 3 interacting protein 1; fam82a1; regulator of G-protein signaling 10; translocase of inner mitochondrial membrane 9 homolog; protein phosphatase 3 , regulatory subunit $\mathrm{B}, \alpha$; ISL LIM homeobox 1; leucine-zipper-like transcription regulator 1; and Pellino E3 ubiquitin protein ligase family member 3) $(\mathrm{P}<0.001)$, and 7 significantly decreased [haptoglobin-related protein; coiled-coil domain containing 124; serpin peptidase inhibitor, clade A ( $\alpha-1$ antiproteinase, antitrypsin), member 1; C11orf46, fibrinogen $\beta$ chain; $\alpha 2$-macroglobulin; and clathrin, light chain $\mathrm{B}](\mathrm{P}<0.001)$. Of the $635 \mathrm{DEPs}$ identified in total, 171 were not detected in the U87 cell lysates, while 40 DEPs were not detected in the TGF- $\beta 1$-induced U87 cell lysates.

Distribution and ontology analysis of identified DEPs. Each of the 635 DEPs (601 genes) was classified according to its cellular localization using data from Swiss-Prot (www.uniprot.org/uniprot), Gene Ontology (GO; geneontology.org), PubMed (www.ncbi.nlm.nih.gov/pubmed) and KEGG (www.genome.jp/kegg). If a protein was detected in more than one cellular compartment, it was additionally taken into consideration. GO analysis demonstrated that cellular localization of DEPs predominantly included cell part (GO:0044464; 38.4\%), organelle (GO:0043226; 27.4\%), macromolecular complex (GO:0032991; 14.0\%), membrane (GO:0016020; 8.0\%), extracellular region (GO:0005576;
8.0\%) and ECM (GO:0031012; 3.6\%). Thus, the majority of the proteins were localized intracellularly.

Proteins were classified according to their cellular biological function, their molecular function and functional class, based on the Protein ANalysis THrough Evolutionary Relationships (PANTHER) proteomic and genomic analysis program (www.pantherdb.org). The majority of DEPs were associated with the following GO terms: Metabolic function (GO:0008152; 28.7\%), cellular processes (GO:0009987; $21.1 \%$ ), localization (GO:0051179; 10.8\%), biological regulation (GO:0065007; 10.5\%), cellular component organization or biogenesis (GO:0071840; 6.5\%), development (GO:0032502; $6.3 \%$ ) and response to stimulus (GO:0050896; 5.5\%).

The majority of DEPs were associated with the following GO functions: Catalytic (GO:0003824; 33.6\%), binding (GO:0005488; 31,6\%), structural molecule (GO:0005198; $10.1 \%$ ), enzyme regulator (GO:0030234; 8.8\%), transporter activity (GO:0005215; 5.0\%), nucleic acid binding transcription factor $(\mathrm{GO}: 0001071 ; 4.3 \%)$ and receptor activity (GO:0004872; 4.0\%).

The 635 DEPs were classed in the basic PANTHER groups as follows: Nucleic acid binding (PC00171; 12.7\%); enzyme modulator (PC00095; 10.5\%); cytoskeletal protein (PC00085; 9.2\%); hydrolase (PC00121; 8.6\%); transferase (PC00220; $6.2 \%)$; transporter (PC00227; 4.9\%); signaling molecule (PC00207; 4.5\%); and transcription factor (PC00218; 4.5\%).

Thus, the majority of DEPs were functionally heterogeneous, and classified into various PANTHER groups as follows: Transcriptional factors (PC00218), nuclear factor- $\kappa \mathrm{B} 1$, MYC binding protein, THO complex 2, PDZ and LIM domain 7 (Enigma), x-prolyl aminopeptidase (aminopeptidase P) 1, general transcription factor IIi; cell adhesion molecules (PC00069), neurofascin, CD209, tetraspanin 9 (TSPAN9), TGF- $\beta$ induced, Integrin, $\beta 3$ (ITGB3), calsyntenin 3; cell junction proteins (PC00070), myosin VA, coiled-coil domain containing 150 , myosin, heavy chain 9 , non-muscle, desmoglein 1, myosin VIIA, myosin IC; ECM proteins (PC00102), a disintegrin and metalloproteinase with thrombospondin motifs 1, Collagen, type XV, $\alpha$ 1,discoidin, CUB And LCCL domain containing 2 , neurexin 2 , laminin, $\beta 1$; kinases (PC00137), $\alpha$-kinase 2, eukaryotic elongation factor-2 kinase, ataxia telangiectasia and Rad3-related protein (ATR), pyridoxine, vitamin B6, diacylglycerol kinase $\alpha$; proteases (PC00190), influenza virus NS1A binding protein, lectin galactoside-binding soluble 3 binding protein, Rho guanine nucleotide exchange factor 10, lysyl oxidase homolog 2, Kell blood group glycoprotein; and signaling molecules (PC00207), TSPAN9, SH3-domain kinase binding protein 1, S100 calcium binding protein A13, fibrinogen $\gamma$ chain, S100 calcium binding protein $\mathrm{P}$; transmembrane receptor regulatory/adaptor protein (PC00226), neuroplastin.

The proteins with increased expression levels in TGF- $\beta 1$-stimulated U87 cells were associated with PANTHER groups including nucleic acid binding (PC00171; 12.6\%), cytoskeletal proteins (PC00085, 10.4\%), enzyme modulators (PC00095; 9.4\%), transferase (PC00220; 7.0\%), transporter (PC00227; 5.4\%) and membrane trafficking proteins (PC00150; $4.8 \%$ ). The majority of proteins with decreased expression levels were part of the PANTHER groups including enzyme modulator (PC00095; 14.8\%), nucleic acid binding (PC00171; 
$13.3 \%$ ), hydrolase (PC00121; 10.2\%), signaling molecule (PC00207; 7.8\%), transcription factor (PC00218; 7.8\%) and oxidoreductase (PC00176; 6.3\%).

A number of the enriched GO terms for the DEPs were associated with the following notable signaling pathways: Cell cycle; integrin signaling pathway; ubiquitin proteasome pathway; inflammation mediated by chemokine and cytokine signaling pathway; Wnt signaling pathway; Notch signaling pathway; angiogenesis; apoptosis signaling pathway; cadherin signaling pathway; epidermal growth factor receptor signaling pathway; fibroblast growth factor (FGF) signaling pathway; platelet-derived growth factor (PDGF) signaling pathway; adherens junctions; cell adhesion molecules; pathways in cancer; and insulin signaling pathway.

\section{Discussion}

The development of malignant glioma proceeds through several stages, including transformation of healthy cells, activation of cell proliferation signals, loss of cell cycle control, development of an invasive phenotype, increased angiogenesis and additional development of clones (20). GBM is the most aggressive type of brain tumor and is characterized by diffuse infiltration of brain parenchyma, aberrant proliferation, radio- and chemotherapy resistance, and relapse following surgical resection $(20,21)$. Advances in molecular technologies, including high-density chip microarrays and genome sequencing, have enabled the evaluation of genetic and epigenetic changes in these tumors at the genome level (22).

Currently, there is no evidence demonstrating that the TGF- $\beta$ signaling pathway has anti-oncogenic activity in glioma, whereas in carcinomas, senescence is induced via TGF- $\beta$ signaling (9). However, in malignant brain tumors, TGF- $\beta$ expression has been previously demonstrated to promote tumor cell survival, and to increase cell proliferation, migration, invasion, angiogenesis, stem cell functioning and immune system suppression (23).

In glioma, TGF- $\beta$ is released from glioma cells via autocrine mechanisms or is secreted from microglial cells. Autocrine secretion of TGF- $\beta$ has been observed in cell lines and cells that have been obtained from surgically removed malignant gliomas $(24,25)$.

Out of 2,555 identified proteins, the current study detected that 635 exhibited significantly altered expression following TGF- $\beta 1$ treatment of U87 cells. The majority of bioinformatic analysis was performed on these proteins, although certain analyses were performed on the entire list of proteins in order to gain a complete picture.

TGF- $\beta$ signaling stimulates a cytostatic response $(26,27)$, which is why proteins involved in cell cycle regulation were analyzed in the present study (Table I). TGF- $\beta 1$ treatment caused a significant decrease in the protein levels of ATR serine/threonine kinase, cell division cycle 16, minichromosome maintenance complex component 5 (MCM5) and MCM6 and an increase in cullin 1. Controlling the levels of cyclin-dependent kinases (CDKs) and their activation is an important mechanism of the anti-proliferative effect of TGF- $\beta$ (28). As Table I demonstrates, the expression levels of CDK9 and CDK inhibitor 2D interacting protein were increased and the CDK5 regulatory subunit associated protein 1-like 1 regulatory subunit was decreased.

Integrins are heterodimeric transmembrane cellular adhesion receptors that interact with various extracellular ligands (for example ECM proteins, including collagen, laminins, vitronectins and fibronectins). These interactions activate integrins and, thus, regulate tumor cell invasion, metastasis, migration, proliferation, angiogenesis and survival $(29,30)$. This demonstrates the importance of integrins in GBM oncogenesis (31). Immunohistological analysis previously demonstrated that integrin $\alpha 3$ (ITGA3) is expressed in the plasma membrane of GBM cells, particularly in invading cells and the surrounding blood vessels (21). The increased expression of ITGA3 enhances migration and invasion of glioma cells and glioblastoma stem cells (GSCs) (32). The current study detected that the expression of various proteins associated with EMT was increased following TGF- $\beta 1$ treatment of U87 cells, including integrins (ITGB1, ITGB3, ITGAV, ITGA5, ITGAX, ITGA3, ITGA8) and fibronectin 1 (FN1) (Table II). It is necessary to note that ITGA8 was only detected in the TGF- $\beta 1$-stimulated cells. ITGA 2 and ITGB3 levels were increased by 2 -fold. FN1 expression was increased 3.78-fold.

The significance of epigenetic alterations in cancer is well-established (25). Histone deacetylases (HDACs) are able to alter chromatin structure to organize DNA and regulate gene transcription in malignant glioma (27). HDAC inhibitors have previously been used to inhibit growth and induce apoptosis of cancer cells (27). Histone acetyltransferases (HATs) and histone hyperacetylation may cause degradation of hypoxia inducible factor- $1 \alpha$ and reduce the level of vascular endothelial growth factor (VEGF), resulting in anti-angiogenic effects (33). In a previous study, treatment with HDACs decreased proliferation of glioblastoma-derived cells and caused their differentiation (34). Analysis of the data of the present study demonstrated that HDAC1 and HDAC2 protein levels were increased (1.52- and 1.69-fold, respectively) and HAT1 levels were decreased (0.67-fold) in TGF- $\beta 1$-stimulated U87 cells.

Heat shock proteins (Hsp), particularly Hsp90, are required for malignant transformation, growth stimulation, survival and invasiveness of cancer cells $(27,35)$. They stabilize the expression of cell surface proteins epidermal growth factor receptor (EGFR)VIII and EGFRvIV, and may promote GBM invasiveness (35). In the present study, TGF- $\beta 1$ stimulation marginally increased Hsp90b1, Hsp90AB1 and Hsp90AA1 expression; however, the effect was not significant. The most substantial increase was observed in Hsp90AA4P (1.46-fold).

The effect of 14-3-3 proteins on cancer development and their interaction with various oncogenes and tumor suppressor genes is complex (36). A previous study investigated the increased immune reactivity of various 14-3-3 isoforms in human astrocytoma samples, and the immunoreactivity of cells was significantly enhanced with increased disease progression (37). Furthermore, $14-3-3 \beta$ and $h$ were identified as two tumor-specific isoforms of 14-3-3 in astrocytomas, and they may be potential candidates for targeted therapies (38). However, the exact function of 14-3-3 proteins in GBM oncogenesis remains to be elucidated. In the present study, the protein expression levels of tyrosine 3-monooxygenase/tryptophan 5-monooxygenase activation protein $\theta$ (YWHAQ), YWHA $\zeta$, YWHA $\gamma$ 
Table I. Changes in expression of cell-cycle proteins following TGF- $\beta 1$ treatment of U87 cells.

\begin{tabular}{llr}
\hline Abbreviation & \multicolumn{1}{c}{ Protein name } & Ratio TGF $\beta /$ control \\
\hline ATR & $\begin{array}{l}\text { Ataxia telangiectasia and Rad3 related; similar to ataxia telangiectasia } \\
\text { and Rad3 related protein }\end{array}$ & 0.12 \\
CDC16 & Cell division cycle 16 homolog (S. cerevisiae) & 0.23 \\
CUL1 & Cullin 1 & 2.53 \\
MCM5 & Minichromosome maintenance complex component 5 & 0.36 \\
MCM6 & Minichromosome maintenance complex component 6 & 0.64 \\
CDK9 & Cyclin-dependent kinase 9 & 1.96 \\
CDKN2D & Cyclin-dependent kinase inhibitor 2D (p19, inhibits CDK4) & 0.97 \\
CDKN2AIP & CDKN2A interacting protein & $0 \uparrow$ \\
CDKAL1 & CDK5 regulatory subunit associated protein 1-like 1 & 0.50 \\
\hline
\end{tabular}

Arrows indicate an increase in expression when the protein was not expressed in the control group. TGF, transforming growth factor.

Table II. Changes in expression of integrins and fibronectin following TGF- $\beta 1$ treatment of U87 cells.

\begin{tabular}{llc}
\hline Abbreviation & Protein name & Ratio TGF $\beta /$ control \\
\hline ITGB2 & Integrin, $\beta 2$ & 1.19 \\
ITGB3 & Integrin, $\beta 3$ & 2.02 \\
ITGB1 & Integrin, $\beta 1$ & 1.55 \\
ITGAV & Integrin, $\alpha \mathrm{V}$ & 1.51 \\
ITGA5 & Integrin, $\alpha 5$ & 1.50 \\
ITGA3 & Integrin, $\alpha 3$ & 0.78 \\
ITGAX & Integrin, $\alpha X$ & 1.52 \\
ITGA8 & Integrin, $\alpha 8$ & $\uparrow \uparrow \uparrow$ \\
ITGA2 & Integrin, $\alpha 2$ & 1.89 \\
FN1 & Fibronectin 1 & 3.78 \\
\hline
\end{tabular}

TGF, transforming growth factor.

and YWHA $\beta$ increased marginally following TGF- $\beta$ treatment of U87 cells. The most substantial increases were observed in YWHA $\eta$ (1.38-fold) and YWHAQ (1.46-fold).

To improve the understanding of underlying biological processes and signaling pathways involved in TGF- $\beta$-induced responses, comprehensive bioinformatic analysis was performed using the Kyoto Encyclopedia of Genes and Genomes (KEGG; www.genome.jp/kegg/) for pathway analysis and GO for biological processes. The descriptions of processes identified as potentially involved in EMT were extensive (39). The KEGG and GO search terms were 'intercellular contacts', 'focal adhesion' and 'actin cytoskeleton'.

Breakdown of intracellular tight junctions during EMT is accompanied by decreased expression of claudin and occludin, and diffusion of zonula occludens 1 [termed tight junction protein 1 (TJP1)] from intercellular contacts $(40,41)$. Following TGF- $\beta 1$ treatment, the TJP1 expression level was increased (1.86-fold) and TJP2 was decreased (0.54-fold). EMT additionally stimulates the expression of neural cell adhesion molecules, which interact with proto-oncogene $\mathrm{Src}$ family tyrosine kinase Fyn (FYN) to reduce inhibition of focal adhesion, migration and invasion (42). In the current study, the FYN protein expression levels were increased 1.45-fold. ECM remodeling and changes in cellular interactions with the ECM are important for the initiation of EMT (35). Integrin complexes enable cells to receive signals from ECM proteins through interaction with signal mediators, including integrin-linked kinase (ILK) and parvin (41,43). Following treatment of U87 cells with TGF- $\beta 1$ for $72 \mathrm{~h}$, there was a slight increase in ILK expression (1.2-fold) and no detectable change in parvin- $\alpha$ levels, whereas the protein level of parvin- $\beta$ was decreased (0.66-fold). Changes in the expression of integrins during EMT are correlated with increased expression of proteases, including matrix metalloproteinase 2 (MMP2) and MMP9, which increases ECM degradation and promotes invasion (44). In the present study, a significant increase in MMP2 expression was detected (2.85-fold), whereas MMP14 levels did not change. In addition to the traditional SMAD-mediated TGF- $\beta$ signaling, TGF- $\beta$ induces signal transduction via Rho GTPases, phosphatidylinositol-4,5-bisphosphate 3-kinase and the mitogen-activated protein kinase signaling pathways $(45,46)$ that also promote EMT $(47-49)$.

Activation of Rho, Rac and cell division control protein 42 homolog GTPases results in reorganization of actin, as well as lamellipodia and filopodia formation (50). A previous study demonstrated that TGF- $\beta$ stimulates partitioning defective 6 homolog family cell polarity regulator via TGF- $\beta$ receptor II and recruits SMAD specific E3 ubiquitin protein ligase 1, which promotes RhoA ubiquitination and degradation when tight junctions are broken (51). TGF- $\beta$ additionally induces RhoA activation (52), partially via promotion of the expression of guanine nucleotide exchange factors, and Rho-associated coiled-coil containing protein kinase 1 and LIM domain kinase 1 activation $(52,53)$. Therefore, decreased expression of RhoA or chemical inhibition of RhoA prevents actin reorganization, which is required for TGF- $\beta$-induced EMT (52-54). The results of the comparative expression analysis of proteins participating in these processes are presented in Table III.

FGF signaling enhances mesenchymal features in epithelial cells (55). In the current study, the levels of FGF18 protein were increased (1.89-fold) in the TGF- $\beta$ stimulated U87 cells. EGF induces endocytosis of E-cadherin in vitro, similar to the 
Table III. Changes in expression of proteins participating in modulation of TGF- $\beta$ signaling during epithelial-to-mesenchymal transition in U87 cells, which are not connected with SMAD proteins.

\begin{tabular}{llc}
\hline Abbreviation & \multicolumn{1}{c}{ Protein name } & Ratio TGFß/control \\
\hline RhoA & Ras homolog gene family, member A & 1.45 \\
RHOC & Ras homolog gene family, member C & 1.19 \\
RAC1 & Ras-related C3 botulinum toxin substrate 1 (rho family, small & 0.84 \\
& GTP binding protein Rac1) & 0.70 \\
RAC2 & Ras-related C3 botulinum toxin substrate 2 (rho family, small & 0.68 \\
& GTP binding protein Rac2) & 0.88 \\
CDC42 & Cell division cycle 42 (GTP binding protein, 25 kDa) & 1.38 \\
CDC42BPB & CDC42 binding protein kinase beta (dystrophia myotonica-protein kinase-like) & \\
ROCK2 & Rho-associated, coiled-coil containing protein kinase 2 & \\
\hline
\end{tabular}

TGF, transforming growth factor; GTP, guanosine-5'-triphosphate.

Table IV.Changes in expression of extracellularmatrix-receptor interaction proteins following TGF- $\beta 1$ treatment of U87 cells.

Ratio TGF $\beta /$

\begin{tabular}{llc} 
Abbreviation & \multicolumn{1}{c}{ Protein name } & control \\
\hline COL1A2 & Collagen, type I, $\alpha 2$ & 3.59 \\
FN1 & Fibronectin 1 & 3.78 \\
HMMR & Hyaluronan-mediated & $\uparrow \uparrow$ \\
& motility receptor (RHAMM) & \\
ITGA8 & Integrin, $\alpha 8$ & $\uparrow \uparrow$ \\
LAMB1 & Laminin, $\beta$ 1 & 7.22 \\
THBS1 & Thrombospondin 1 & $\uparrow \uparrow$ \\
\hline
\end{tabular}

Arrows indicate an increase in expression when the protein was not expressed in the control group. TGF, transforming growth factor; RHAMM, receptor for hyaluronan mediated motility.

effect of SNAIL family transcriptional repressor 1 or TWIST family bHLH transcription factor 1 , which decrease the levels of E-cadherin $(56,57)$. EGF also activates EMT in epithelial explants, resulting in increased mobility of cells and MMP2and MMP9-mediated proteolysis, which is dependent on ILK and the extracellular signal-regulated kinase/mitogen-activated protein kinase signaling pathways (58). Activation of human EGF2 receptor (human epidermal growth factor 2; also termed ERBB2) in the epithelial cells of the mammary gland causes development of tumors with an EMT phenotype, which are able to escape immune system responses $(59,60)$. In the current study, stimulation with TGF- $\beta$ induced a significant increase in ERBB2 interactive protein expression (4.79-fold). A previous report demonstrated that PDGF induces breakdown of adhesive cellular contacts, nuclear localization of $\beta$-catenin and E-cadherin level repression in colon adenocarcinoma cells (61). Ablation of the PDGF-receptor $\alpha$-subunit enhances the formation and migration of mesenchymal-like cells, and decreases MMP2 activity (62). VEGF is understood to be associated with PDGF and induces angiogenesis, which also causes EMT (63).
As previously demonstrated, TGF- $\beta$ stimulates glioma growth via interactions with the PDGF- $\beta$ signaling pathway (64). TGF- $\beta$ is able to increase expression levels of PDGF- $\beta$ mRNA and protein, and enhance the phosphorylation of the PDGF receptor; thus, increasing proliferation of U373MG glioblastoma cells (65). The results of the current study demonstrated that the expression of PDGF receptor $\beta$ was significantly increased (1.74-fold) following TGF- $\beta 1$ treatment of U87 cells, whereas the levels of PDGF receptor $\alpha$ were not significantly altered by TGF- $\beta 1$ treatment (1.1-fold increase).

Bioinformatic analysis demonstrated a slight annotation enrichment of proteins involved in ECM-receptor interaction (Table IV), regulation of actin cytoskeleton, the spliceosome (Table V), DNA replication (Table VI), adherens or tight junctions and focal adhesion, which are established signaling responses to TGF- $\beta(10,66-68)$. Proteins in these signaling pathways may be potential markers of metastasis and targets for glioblastoma treatment. Additional annotations corresponded to functions that are not typically associated with TGF- $\beta$ signal transduction, for example DNA mismatch repair proteins (Table VII).

Cellular adhesion receptors are highly expressed in GBM cells and are important for their invasion. Glioma cells use these receptors for adhesion and migration via interaction with components of the ECM, which is specifically distributed and regulated within the human brain and spinal cord. Therefore, glioma cell invasion into adjacent brain tissue depends on the interaction between glioma cells and the ECM (69).

TGF- $\beta$ positively regulates the expression of ECM components, including type I collagen and FN1, which are significant proteins involved in tumorigenesis (70). As Table VI demonstrates, TGF- $\beta 1$ impact on U87 cells increases collagen, type I, $\alpha 2$ and FN1 expression 3.59- and 3.78-fold, respectively. In pathological cases, intensified TGF- $\beta$ signaling may cause excessive accumulation of ECM (71). During EMT, cells obtain mesenchymal markers, including FN1 and vimentin, with the levels of vimentin observed to be non-significantly increased in the current study, unlike the levels of FN1.

The ECM of the central nervous system is enriched by hyaluronate (HA). CD44 and receptor for HA-mediated motility 
Table V. Changes in expression of spliceosome proteins following TGF- $\beta 1$ treatment of U87 cells.

\begin{tabular}{llc}
\hline Abbreviation & \multicolumn{1}{c}{ Protein name } & Ratio TGF $\beta /$ control \\
\hline LSM2 & LSM2 homolog, U6 small nuclear RNA associated (S. cerevisiae) & $\uparrow \uparrow$ \\
PRPF19 & PSO4 pre-mRNA processing factor 19 homolog (S. cerevisiae) & 5.95 \\
WBP11 & WW domain binding protein 11 & 2.99 \\
NCBP1 & Nuclear cap binding protein subunit 1, 80 kDa & 3.29 \\
SFRS2 & Splicing factor, arginine/serine-rich 2 & 3.64 \\
SMNDC1 & Survival motor neuron domain containing 1 & \\
TXNL4A & Thioredoxin-like 4A & $\uparrow \uparrow$ \\
\hline
\end{tabular}

Arrows indicate an increase in expression when the protein was not expressed in the control group. TGF, transforming growth factor.

Table VI. Changes in expression of DNA replication proteins following TGF- $\beta 1$ treatment of U87 cells.

\begin{tabular}{llc}
\hline Abbreviation & \multicolumn{1}{c}{ Protein name } & $\begin{array}{c}\text { Ratio TGF } \beta \text { / } \\
\text { control }\end{array}$ \\
\hline 1POLE2 & $\begin{array}{l}\text { Polymerase (DNA directed), } \\
\text { Epsilon 2 (p59 subunit) }\end{array}$ & $\uparrow \uparrow$ \\
RPA2 & $\begin{array}{l}\text { Replication protein A2,32 kDa } \\
\text { RPA3 }\end{array}$ & $\begin{array}{l}\uparrow \uparrow \\
\text { Replication protein A3, 14 kDa }\end{array}$ \\
\hline
\end{tabular}

Arrows indicate an increase in expression when the protein was not expressed in the control group. TGF, transforming growth factor.

Table VII. Changes in expression of DNA mismatch repair proteins following TGF- $\beta 1$ treatment of U87 cells.

\begin{tabular}{llc}
\hline Abbreviation & \multicolumn{1}{c}{ Protein name } & $\begin{array}{c}\text { Ratio TGF } \beta \text { / } \\
\text { control }\end{array}$ \\
\hline MLH3 & mutL homolog 3 & $\uparrow \uparrow$ \\
MSH6 & mutS homolog 6 & $\uparrow \uparrow$ \\
RPA2 & Replication protein A2, 32 kDa & $\uparrow \uparrow$ \\
Rpa3 & Replication protein A3, 14 kDa & 3.21 \\
\hline
\end{tabular}

Arrows indicate an increase in expression when the protein was not expressed in the control group. TGF, transforming growth factor.

(RHAMM) are ubiquitous receptors for HA. RHAMM mediates migration and proliferation of glioma cells, with the levels of RHAMM increasing with the progression of disease (72). The current study only detected RHAMM expression in TGF- $\beta 1$-stimulated U87 cells, while the levels of CD44 did not change during the experiment.

ECM remodeling via ECM protein proteolysis is an important step in the metastatic process that enables neoplastic cells to invade through the basal lamina (BL) into the stroma (73). Proteolysis stimulates signaling pathways that regulate migratory, functional or signaling molecules, including growth factors, and the generation of biologically active fragments of the ECM (74). Laminins are ECM glycoprotein components present in the BL and have been previously identified as substrates for cell migration and adhesion (75). To the best of our knowledge, the function of laminin $\beta 1$ in these processes has not been previously investigated. As demonstrated in Table IV, the levels of laminin $\beta 1$ were increased significantly (7.22-fold) in TGF- $\beta 1$-induced U87 cells.

Angiogenesis is crucial for supporting the growth and metastasis of tumors $(27,41)$. Thrombospondin-1 (TSP-1) is a powerful inhibitor of angiogenesis, and negative regulation of TSP-1 may alter tumor growth by modulating angiogenesis in various tumor types. TSP-1 expression is positively regulated by tumor-suppressing gene $\mathrm{p} 53$, and negatively regulated by oncogenes, including Myc and Ras. TSP-1 reduces angiogenesis by inhibiting migration and proliferation of endothelial cells and inducing apoptosis. Furthermore, TSP-1 activation of TGF- $\beta 1$ is important for the regulation of tumor progression. Investigating the molecular mechanisms of TSP-1-mediated suppression of angiogenesis and tumor progression may aid the development of novel diagnostic methods and cancer therapeutics (76). In the present study, TSP-1 was only detected following TGF- $\beta 1$ treatment of glioblastoma cells (Table IV).

Gliomas have a subpopulation of cells that exhibit stem-like properties. This population of cells has been suggested to be involved in the initiation, metastasis, support and relapse of gliomas. Treatment methods that attempt to regulate GSC function may improve survival rates for patients with glioma $(77,78)$.

The TGF- $\beta$ superfamily is crucial for morphogenesis and the specification of cell progenies during the development of the human brain $(79,80)$. Ikushima et al (7) demonstrated that autocrine TGF- $\beta$ signaling is an important factor in supporting the stem cell-like phenotype of GSCs. The association between TGF- $\beta$ and stem cell properties was also demonstrated in mammary gland epithelium, where a short-term incubation of mammary epithelial cells with TGF- $\beta$ activated EMT and increased the ability of the cells to form mammospheres (81). Similarly, incubation with TGF- $\beta$ increased the formation of neurospheres in a primary culture of brain tumor cells, demonstrating that TGF- $\beta$ increases the self-restoration ability of GSCs (82).

Attempts to develop targeted therapies for GBM are predominantly focused on the analysis of GSCs. Previous studies have characterized the spliceosome proteins that are specifically required for GSC growth and survival compared 
with neural stem cells and other types of non-transformed cells $(47,72,82)$. As demonstrated in Table V, TGF- $\beta 1$ actively modulates the expression of certain spliceosomal proteins of this group in U87 cells. The protein expression of pre-mRNA processing factor 19, WW domain binding protein 11, nuclear cap binding protein subunit 1 and serine/arginine-rich splicing factor 2 was increased. Notably, LSM2 homolog U6 small nuclear RNA, mRNA degradation associated protein, survival motor neuron domain containing 1 and thioredoxin like $4 \mathrm{~A}$ proteins were only detected in the lysates of TGF- $\beta 1$-treated U87 cells.

In conclusion, the current study investigated the underlying molecular mechanisms that mediate the effect of TGF- $\beta 1$ on U87 human glioblastoma cells. The intracellular processes identified to be involved in the regulation of malignant glioma oncogenesis by TGF- $\beta 1$ included EMT, ECM-receptor interaction, regulation of the actin cytoskeleton, spliceosomal functions, DNA replication, adherens or tight junctions and focal adhesions, with significant patterns being discovered. The current study used comparative proteome mapping to identify candidate markers of glioblastoma metastasis and potential targets for glioma therapeutics.

TFG-1 $\beta$ changes the molecular phenotype of human glioblastoma cells. In response to TFG-1 $\beta$, the expression of 512 proteins associated with survival, proliferation, cell migration and DNA repair is increased. In addition, the expression of 123 proteins responsible for apoptosis, interaction with the extracellular matrix and aerobic metabolism is decreased. Therefore, TFG- $1 \beta$ holds a critical role in glial brain tumor biology and is among the key stimulators of GBM invasive growth. This makes TFG-1 $\beta$ a promising target for targeted cancer therapy. Since tumor stem cells are tightly involved in GBM cancerogenesis, future studies should be focused on the impact TFG-1 $\beta$ has on various subpopulations of this cell type.

\section{Acknowledgements}

The current study was funded by the Ministry of Education and Science of the Russian Federation (grant no., 14.575.21.0038; ID code, RFMEF157514X0038).

\section{References}

1. Holland EC: Glioblastoma multiforme: The terminator. Proc Natl Acad Sci USA 97: 6242-6244, 2000.

2. Adamson C, Kanu OO, Mehta AI, Di C, Lin N, Mattox AK and Bigner DD: Glioblastoma multiforme: A review of where we have been and where we are going. Expert Opin Investig Drugs 18: 1061-1083, 2009.

3. Wang $\mathrm{Y}$ and Jiang $\mathrm{T}$ : Understanding high grade glioma: Molecular mechanism, therapy and comprehensive management. Cancer Lett 331: 139-146, 2013.

4. Westphal M and Lamszus K: The neurobiology of gliomas: From cell biology tothe development of therapeutic approaches. Nat Rev Neurosci 12: 495-508, 2011.

5. Rich JN: The role of transforming growth factor-beta in primary brain tumors. Front Biosci 8: e245-e260, 2003.

6. Platten M, Wick W and Weller M: Malignant glioma biology: Role for TGF-beta in growth, motility, angiogenesis, and immune escape. Microsc Res Tech 52: 401-410, 2001.

7. Ikushima H, Todo T, Ino Y, Takahashi M, Miyazawa K and Miyazono K: Autocrine TGF-beta signaling maintains tumorigenicity of glioma-initiating cells through Sry-related HMG-box factors. Cell Stem Cell 5: 504-514, 2009.
8. Gregory PA, Bracken CP, Smith E, Bert AG, Wright JA, Roslan S, Morris M, Wyatt L, Farshid G, Lim YY, et al: An autocrine TGF-beta/ZEB/miR-200 signaling network regulates establishment and maintenance of epithelial-mesenchymal transition. Mol Biol Cell 22: 1686-1698, 2011

9. Seoane J: Escaping from the TGFbeta anti-proliferative control. Carcinogenesis 27: 2148-2156, 2006.

10. Massagué J: TGFbeta in cancer. Cell 134: 215-230, 2008.

11. Derynck R, Akhurst RJ and Balmain A: TGF-beta signaling in tumor suppression and cancer progression. Nat Genet 29: 117-129, 2001.

12. Siegel PM and Massagué J: Cytostatic and apoptotic actions of TGF-beta in homeostasis and cancer. Nat Rev Cancer 3: 807-821, 2003.

13. Rahimi RA and Leof EB: TGF-beta signaling: A tale of two responses. J Cell Biochem 102: 593-608, 2007.

14. Massagué $J$ and Gomis RR: The logic of TGFbeta signaling. FEBS Lett 580: 2811-2820, 2006.

15. Kalluri R and Weinberg RA: The basics of epithelial-mesenchymal transition. J Clin Invest 119: 1420-1428, 2009.

16. Morel AP, Lièvre M, Thomas C, Hinkal G, Ansieau S and Puisieux A: Generation of breast cancer stem cells through epithelial-mesenchymal transition. PLoS One 3: e2888, 2008.

17. Taube JH, Herschkowitz JI, Komurov K, Zhou AY, Gupta S, Yang J, Hartwell K, Onder TT, Gupta PB, Evans KW, et al: Core epithelial-to-mesenchymal transition interactome gene-expression signature is associated with claudin-low and metaplastic breast cancer subtypes. Proc Natl Acad Sci USA 107: 15449-15454, 2010.

18. Bryukhovetskiy A, Shevchenko V, Kovalev S, Chekhonin V, Baklaushev V, Bryukhovetskiy I and Zhukova M: To the novel paradigm of proteome-based cell therapy of tumors: Through comparative proteome mapping of tumor stem cells and tissue-specific stem cells of humans. Cell Transplant 23 (Suppl 1): S151-S170, 2014.

19. Cox J and Mann M: MaxQuant enables high peptide identification rates, individualized p.p.b.-range mass accuracies and proteome-wide protein quantification. Nat Biotechnol 26: 1367-1372, 2008

20. Louis DN: Molecular pathology of malignant gliomas. Annu Rev Pathol 1: 97-117, 2006.

21. Louis DN, Ohgaki H, Wiestler OD, Cavenee WK, Burger PC, Jouvet A, Scheithauer BW and Kleihues P: The 2007 WHO classification of tumours of the central nervous system. Acta Neuropathol 114: 97-109, 2007.

22. Cancer Genome Atlas Research Network: Comprehensive genomic characterization defines human glioblastoma genes and core pathways. Nature 455: 1061-1068, 2008.

23. Platten M, Wick W and Weller M: Malignant glioma biology: Role for TGF-beta in growth, motility, angiogenesis, and immune escape. Microsc Res Tech 52: 401-410, 2001.

24. Sasaki A, Naganuma H, Satoh E, Nagasaka M, Isoe S, Nakano S and Nukui $\mathrm{H}$ : Secretion of transforming growth factor-beta 1 and -beta 2 by malignant glioma cells. Neurol Med Chir (Tokyo) 35: 423-430, 1995.

25. Wesolowska A, Kwiatkowska A, Slomnicki L, Dembinski M Master A, Sliwa M, Franciszkiewicz K, Chouaib S and Kaminska B: Microglia-derived TGF-beta as an important regulator of glioblastoma invasion - an inhibition of TGF-beta-dependent effects by shRNA against human TGF-beta type II receptor. Oncogene 27: 918-930, 2008.

26. Hannon GJ and Beach D: P15INK4B is a potential effector of TGF-beta-induced cell-cycle arrest. Nature 371: 257-261, 1994.

27. Datto MB, Li Y, Panus JF, Howe DJ, Xiong Y and Wang XF: Transforming growth factor beta induces the cyclin-dependent kinase inhibitor p21 through a p53-independent mechanism. Proc Natl Acad Sci USA 92: 5545-5549, 1995.

28. Eblen ST, Fautsch MP, Burnette RJ, Joshi P and Leof EB: Cell cycle-dependent inhibition of $\mathrm{p} 34 \mathrm{cdc} 2$ synthesis by transforming growth factor beta 1 in cycling epithelial cells. Cell Growth Differ 5: 109-116, 1994.

29. Ignotz RA, Endo T and Massagué J: Regulation of fibronectin and type I collagen mRNA levels by transforming growth factor-beta. J Biol Chem 262: 6443-6446, 1987.

30. Margadant $\mathrm{C}$ and Sonnenberg A: Integrin-TGF-beta crosstalk in fibrosis, cancer and wound healing. EMBO Rep 11: 97-105, 2010.

31. Nakada M, Nakada S, Demuth T, Tran NL, Hoelzinger DB and Berens ME: Molecular targets of glioma invasion. Cell Mol Life Sci 64: 458-478, 2007. 
32. Nakada M, Nambu E, Furuyama N, Yoshida Y, Takino T, Hayashi Y, Sato H, Sai Y, Tsuji T, Miyamoto KI, et al: Integrin $\alpha 3$ is overexpressed in glioma stem-like cells and promotes invasion. Br J Cancer 108: 2516-2524, 2013.

33. Galanis E, Jaeckle KA, Maurer MJ, Reid JM, Ames MM, Hardwick JS, Reilly JF, Loboda A, Nebozhyn M, Fantin VR, et al: Phase II trial of vorinostat in recurrent glioblastoma multiforme: A north central cancer treatment group study. J Clin Oncol 27: 2052-2058, 2009.

34. Alvarez AA, Field M, Bushnev S, Longo MS and Sugaya K: The effect of histone deacetylase inhibitors on glioblastoma-derived stem cells. J Mol Neurosci 55: 7-20, 2015.

35. Pines G, Huang PH,Zwang Y, White FM and Yarden Y: EGFRvIV: A previously uncharacterized oncogenic mutant reveals a kinase autoinhibitory mechanism. Oncogene 29: 5850-5860, 2010

36. Liang S, Shen G, Liu Q, Xu Y, Zhou L, Xiao S, Xu Z, Gong F, You $\mathrm{C}$ and Wei Y: Isoform-specific expression and characterization of 14-3-3 proteins in human glioma tissues discovered by stable isotope labeling with amino acids in cell culture-based proteomic analysis. Proteomics Clin Appl 3: 743-753, 2009.

37. Cao L, Cao W, Zhang W, Lin H, Yang X, Zhen H, Cheng J, Dong $\mathrm{W}$, Huo $\mathrm{J}$ and Zhang X: Identification of 14-3-3 protein isoforms in human astrocytoma by immunohistochemistry. Neurosci Lett 432: 94-99, 2008.

38. Yang X, Cao W, Lin H, Zhang W, Lin W, Cao L, Zhen H, Huo J and Zhang X: Isoform-specific expression of 14-3-3 proteins in human astrocytoma. J Neurol Sci 276: 54-59, 2009.

39. Lamouille S, Xu J and Derynck R: Molecular mechanisms of epithelial-mesenchymal transition. Nat Rev Mol Cell Biol 15: 178-196, 2014

40. Huang RY, Guilford P and Thiery JP: Early events in cell adhesion and polarity during epithelial-mesenchymal transition. J Cell Sci 125: 4417-4422, 2012

41. Yilmaz M and Christofori G: EMT, the cytoskeleton, and cancer cell invasion. Cancer Metastasis Rev 28: 15-33, 2009.

42. Lehembre F, Yilmaz M, Wicki A, Schomber T, Strittmatter K, Ziegler D, Kren A, Went P, Derksen PW, Berns A, et al: NCAM-induced focal adhesion assembly: A functional switch upon loss of E-cadherin. EMBO J 27: 2603-2615, 2008.

43. Hansen SM, Berezin V and Bock E: Signaling mechanisms of neurite outgrowth induced by the cell adhesion molecules NCAM and N-cadherin. Cell Mol Life Sci 65: 3809-3821, 2008.

44. Nisticò P, Bissell MJ and Radisky DC: Epithelial-mesenchyma transition: General principles and pathological relevance with special emphasis on the role of matrix metalloproteinases. Cold Spring Harb Perspect Biol 4: pii: a011908, 2012.

45. Derynck R and Zhang YE: Smad-dependent and Smad-independent pathways in TGF-beta family signalling. Nature 425 : 577-584, 2003

46. Moustakas A and Heldin CH: Non-Smad TGF-beta signals. J Cell Sci 118: 3573-3584, 2005

47. Xu J, Lamouille S and Derynck R: TGF-beta-induced epithelial to mesenchymal transition. Cell Res 19: 156-172, 2009.

48. Zavadil J and Böttinger EP: TGF-beta and epithelial-to-mesenchymal transitions. Oncogene 24: 5764-5774, 2005.

49. Lamouille $S$ and Derynck R: Emergence of the phosphoinositide 3-kinase-Akt-mammalian target of rapamycin axis in transforming growth factor- $\beta$-induced epithelial-mesenchymal transition. Cells Tissues Organs 193: 8-22, 2011

50. Ridley AJ: Life at the leading edge. Cell 145: 1012-1022, 2011.

51. Ozdamar B, Bose R, Barrios-Rodiles M, Wang HR, Zhang Y and Wrana JL: Regulation of the polarity protein Par6 by TGFbeta receptors controls epithelial cell plasticity. Science 307: $1603-1609,2005$.

52. Bhowmick NA, Ghiassi M, Bakin A, Aakre M, Lundquist CA, Engel ME, Arteaga CL and Moses HL: Transforming growth factor-betal mediates epithelial to mesenchymal transdifferentiation through a RhoA-dependent mechanism. Mol Biol Cell 12: 27-36, 2001.

53. Vardouli L, Moustakas A and Stournaras C: LIM-kinase 2 and cofilin phosphorylation mediate actin cytoskeleton reorganization induced by transforming growth factor- $\beta$. J Biol Chem 280: 11448-11457, 2005.

54. Tavares AL, Mercado-Pimentel ME, Runyan RB and Kitten GT: TGF beta-mediated RhoA expression is necessary for epithelial-mesenchymal transition in the embryonic chick heart. Dev Dyn 235: 1589-1598, 2006.

55. Sun X, Meyers EN, Lewandoski M and Martin GR: Targeted disruption of Fgf8 causes failure of cell migration in the gastrulating mouse embryo. Genes Dev 13: 1834- 1846, 1999.
56. Lu Z, Ghosh S, Wang Z and Hunter T: Downregulation of caveolin-1 function by EGF leads to the loss of E-cadherin, increased transcriptional activity of beta-catenin, and enhanced tumor cell invasion. Cancer Cell 4: 499-515, 2003.

57. Lo HW, Hsu SC, Xia W, Cao X, Shih JY, Wei Y, Abbruzzese JL, Hortobagyi GN and Hung MC: Epidermal growth factor receptor cooperates with signal transducer and activator of transcription 3 to induce epithelial-mesenchymal transition in cancer cells via up-regulation of TWIST gene expression. Cancer Res 67: 9066-9076, 2007.

58. Ahmed N, Maines-Bandiera S, Quinn MA, Unger WG, Dedhar S and Auersperg N: Molecular pathways regulating EGF-induced epithelio-mesenchymal transition in human ovarian surface epithelium. Am J Physiol Cell Physiol 290: C1532-C1542, 2006

59. Moody SE, Perez D, Pan TC, Sarkisian CJ, Portocarrero CP, Sterner CJ, Notorfrancesco KL, Cardiff RD and Chodosh LA: The transcriptional repressor Snail promotes mammary tumor recurrence. Cancer Cell 8: 197-209, 2005.

60. Knutson KL, Lu H, Stone B, Reiman JM, Behrens MD, Prosperi CM, Gad EA, Smorlesi A and Disis ML: Immunoediting of cancers may lead to epithelial to mesenchymal transition. J Immunol 177: 1526-1533, 2006.

61. Yang L, Lin C and Liu ZR: P68 RNA helicase mediates PDGF-induced epithelial mesenchymal transition by displacing Axin from beta-catenin. Cell 127: 139-155, 2006.

62. Robbins JR, McGuire PG, Wehrle-Haller B and Rogers SL: Diminished matrix metalloproteinase 2 (MMP-2) in ectomesenchyme-derived tissues of the Patch mutant mouse: Regulation of MMP-2 by PDGF and effects on mesenchymal cell migration. Dev Biol 212: 255-263, 1999.

63. Wanami LS, Chen HY, Peiró S, García de Herreros A and Bachelder RE: Vascular endothelial growth factor-A stimulates Snail expression in breast tumor cells: Implications for tumor progression. Exp Cell Res 314: 2448-2453, 2008.

64. Bruna A, Darken RS, Rojo F, Ocaña A, Peñuelas S, Arias A, Paris R, Tortosa A, Mora J, Baselga J and Seoane J: High TGFbeta-Smad activity confers poor prognosis in glioma patients and promotes cell proliferation depending on the methylation of the PDGF-B gene. Cancer Cell 11: 147-160, 2007.

65. Guo P, Hu B, Gu W, Xu L, Wang D, Huang HJ, Cavenee WK and Cheng SY: Platelet-derived growth factor-B enhances glioma angiogenesis by stimulating vascular endothelial growth factor expression in tumor endothelia and by promoting pericyte recruitment. Am J Pathol 162: 1083-1093, 2003.

66. Lamouille S, Connolly E, Smyth JW, Akhurst RJ and Derynck R: TGF- $\beta$-induced activation of mTOR complex 2 drives epithelial-mesenchymal transition and cell invasion. J Cell Sci 125: 1259-1273, 2012.

67. Kardassis D, Murphy C, Fotsis T, Moustakas A and Stournaras C: Control of transforming growth factor beta signal transduction by small GTPases. FEBS J 276: 2947-2965, 2009.

68. Katsuno Y, Lamouille S and Derynck R: TGF- $\beta$ signaling and epithelial-mesenchymal transition in cancer progression. Curr Opin Oncol 25: 76-84, 2013.

69. Goldbrunner RH, Bernstein JJ and Tonn JC: ECM-mediated glioma cell invasion. Microsc Res Tech 43: 250-257, 1998.

70. Verrecchia F and Mauviel A: Transforming growth factor-beta signaling through the Smad pathway: Role in extracellular matrix gene expression and regulation. J Invest Dermatol 118: 211-215, 2002

71. Schmid P, Itin P, Cherry G, Bi C and Cox DA: Enhanced expression oftransforming growth factor-beta type I and type II receptors in wound granulation tissue and hypertrophic scar. Am J Pathol 152: 485-493, 1998.

72. Akiyama Y, Jung S, Salhia B, Lee S, Hubbard S, Taylor M, Mainprize T, Akaishi K, van Furth W and Rutka JT: Hyaluronate receptors mediating glioma cell migration and proliferation. J Neurooncol 53: 115-127, 2001.

73. Noël A, Gilles C, Bajou K, Devy L, Kebers F, Lewalle JM, Maquoi E, Munaut C, Remacle A and Foidart JM: Emerging roles for proteinasesin cancer. Invasion Metastasis 17: 221-239, 1997.

74. Kleinman HK, Koblinski J, Lee S and Engbring J: Role of basement membrane in tumor growth and metastasis. Surg Oncol Clin N Am 10: 329-338, 2001.

75. Bair EL, Chen ML, McDaniel K, Sekiguchi K, Cress AE, Nagle RB and Bowden GT: Membrane type 1 matrix metalloprotease cleaves laminin-10 and promotes prostate cancer cell migration. Neoplasia 7: 380-389, 2005. 
76. Ren B, Yee KO, Lawler J and Khosravi-Far R: Regulation of tumor angiogenesis by thrombospondin-1. Biochim Biophys Acta 1765: 178-188, 2006.

77. Singh SK, Hawkins C, Clarke ID, Squire JA, Bayani J, Hide T, Henkelman RM, Cusimano MD and Dirks PB: Identification of human brain tumour initiating cells. Nature 432: 396-401, 2004

78. Bao S, Wu Q, McLendon RE, Hao Y, Shi Q, Hjelmeland AB, Dewhirst MW, Bigner DD and Rich JN: Glioma stem cells promote radioresistance by preferential activation of the DNA damage response. Nature 444: 756-760, 2006.

79. Schier AF and Talbot WS: Nodal signaling and the zebrafish organizer. Int J Dev Biol 45: 289-297, 2001.
80. Muñoz-Sanjuán I and Brivanlou AH: Neural induction, the default model and embryonic stem cells. Nat Rev Neurosci 3: 271-280, 2002.

81. Mani SA, Guo W, Liao MJ, Eaton EN, Ayyanan A, Zhou AY, Brooks M, Reinhard F, Zhang CC, Shipitsin M, et al: The epithelial-mesenchymal transition generates cells with properties of stem cells. Cell 133: 704-715, 2008.

82. Peñuelas S, Anido J, Prieto-Sánchez RM, Folch G, Barba I, Cuartas I, García-Dorado D, Poca MA, Sahuquillo J, Baselga J and Seoane J: TGF-beta increases glioma-initiating cell self-renewal through the induction of LIF in human glioblastoma. Cancer Cell 15: 315-327, 2009. 